EPJ Web of Conferences 92,02044 (2015)

DOI: $10.1051 /$ epjconf/ 20159202044

C Owned by the authors, published by EDP Sciences, 2015

\title{
Numerical simulation of the passive gas mixture flow
}

\author{
Martin $\mathrm{Kyncl}^{1, \mathrm{a}}$ and Jaroslav Pelant ${ }^{1, \mathrm{~b}}$ \\ ${ }^{1}$ Výzkumný a Zkušební Letecký Ústav, a.s., VZLÚ, \\ Beranových 130, 19905 Praha - Letňany, Czech Republic
}

\begin{abstract}
We work with the system of equations describing non-stationary compressible turbulent multicomponent flow in the gravitational field, and we focus on the numerical solution of these equations. In these computations we assume the mixture of perfect inert gases. The thermodynamic constants are functions in time and space. The finite volume method is used. In order to solve the local boundary problem at each mesh face, we use the original analysis of the exact solution of the Riemann problem. The roughness of the surface is simulated via the specific dissipation at the wall. We show the computational results obtained with the own-developed code (C,FORTRAN) for the solution of the 3D compressible turbulent mixture flow. The originality of this work lies with the special handling of the boundary conditions, which shows superior behavior, and own computational code.
\end{abstract}

\section{Introduction}

The aim of this work is to numerically simulate complicated behaviour of the perfect gas mixture. In this contribution we consider the Reynolds- Averaged Navier-Stokes equations with the $\mathrm{k}-\omega$ model of turbulence. This system is equipped with the equation of state in more general form, and with the mass conservation of the additional gas specie.

\section{Formulation of the Equations}

We consider the conservation laws for viscous compressible turbulent flow of ideal gas with the zero heat sources in a domain $\Omega \in R^{N}$, and time interval $(0, T)$, with $T>0$. The system of the Reynolds-Averaged Navier-Stokes equations in $3 \mathrm{D}$ has the form

$$
\frac{\partial \boldsymbol{w}}{\partial t}+\sum_{s=1}^{3} \frac{\partial \boldsymbol{f}_{s}(\boldsymbol{w})}{\partial x_{s}}=\sum_{s=1}^{3} \frac{\partial \boldsymbol{R}_{s}(\boldsymbol{w}, \nabla \boldsymbol{w})}{\partial x_{s}}+\boldsymbol{S} \quad \text { in } Q_{T}=\Omega \times(0, T)
$$

Here $x_{1}, x_{2}, x_{3}$ are the space coordinates, $t$ the time, $\boldsymbol{w}=$ $\boldsymbol{w}(x, t)=\left(\varrho, \varrho v_{1}, \varrho v_{2}, \varrho v_{3}, E\right)^{T}$ is the state vector, $\boldsymbol{f}_{s}=$ $\left(\varrho v_{s}, \varrho v_{s} v_{1}+\delta_{s 1} p, \varrho v_{s} v_{2}+\delta_{s 2} p, \varrho v_{s} v_{3}+\delta_{s 3} p,(E+p) v_{s}\right)^{T}$ are the inviscid fluxes, $R_{s}=\left(0, \tau_{s 1}, \tau_{s 2}, \tau_{s 3}, \sum_{r=1}^{3} \tau_{s r} v_{r}+\right.$ $\left.C_{k} \partial \theta / \partial x_{S}\right)^{T}$ are the viscous fluxes, $S$ are additional sources. $\boldsymbol{v}=\left(v_{1}, v_{2}, v_{3}\right)^{T}$ denotes the velocity vector, $\varrho$ is the density, $p$ the pressure, $\theta$ the absolute temperature, $E=\varrho e+\frac{1}{2} \varrho v^{2}$ the total energy. Further $\tau_{i j}=\left\{\begin{array}{ll}\left(\mu+\mu_{T}\right) S_{i j}, & i \neq j \\ \left(\mu+\mu_{T}\right) S_{i j}-\frac{2}{3} \varrho k, & i=j\end{array}\right.$, with $S_{11}=\frac{2}{3}\left(2 \frac{\partial v_{1}}{\partial x_{1}}-\frac{\partial v_{2}}{\partial x_{2}}-\frac{\partial v_{3}}{\partial x_{3}}\right), S_{12}=\frac{\partial v_{1}}{\partial x_{2}}+\frac{\partial v_{2}}{\partial x_{1}}, S_{13}=\frac{\partial v_{1}}{\partial x_{3}}+$ $\frac{\partial v_{3}}{\partial x_{1}}, S_{21}=S_{12}, S_{22}=\frac{2}{3}\left(-\frac{\partial v_{1}}{\partial x_{1}}+2 \frac{\partial v_{2}}{\partial x_{2}}-\frac{\partial v_{3}}{\partial x_{3}}\right), S_{23}=\frac{\partial v_{2}}{\partial x_{3}}+\frac{\partial v_{3}}{\partial x_{2}}$, $S_{31}=S_{13}, S_{32}=S_{23}, S_{33}=\frac{2}{3}\left(-\frac{\partial v_{1}}{\partial x_{1}}-\frac{\partial v_{2}}{\partial x_{2}}+2 \frac{\partial v_{3}}{\partial x_{3}}\right)$, where $\mu$ is the dynamic viscosity coefficient dependent on temperature, $\mu_{T}$ is the eddy-viscosity coefficient. For the specific

\footnotetext{
a e-mail: kyncl@vzlu.cz

b e-mail: pelant@vzlu.cz
}

internal energy $e=c_{v} \theta$ we assume the caloric equation of state $e=p / \varrho(\gamma-1), c_{v}$ is the specific heat at constant volume, $\gamma>1$ is called the Poisson adiabatic constant. The constant $C_{k}$ denotes the heat conduction coefficient $C_{k}=\left(\frac{\mu}{P_{r}}+\frac{\mu_{T}}{P_{r_{T}}}\right) c_{v} \gamma$, and $P_{r}$ is laminar and $P_{r_{T}}$ is turbulent Prandtl constant number. In our application of flow in the gravitational field we set the source terms to $\boldsymbol{S}=\left(0, \varrho g_{1}, \varrho g_{2}, \varrho g_{3}, \varrho \boldsymbol{g} \cdot \boldsymbol{v}\right)$, where $\boldsymbol{g}=\left(g_{1}, g_{2}, g_{3}\right)$ is the gravity vector. For the gas mixture with two species we use the Dalton's law for the total mixture pressure

$$
p=p_{1}+p_{2}
$$

where $p_{1}$ and $p_{2}$ are the partial pressures of the first and second component gas. Let $\varrho_{1}$ and $\varrho_{2}$ be the mass density of these components. Then the total mass density of mixture is

$$
\varrho=\varrho_{1}+\varrho_{2} .
$$

Temperature $\theta$ is same for all gases in the mixture, and the equation of state holds

$$
p_{i}=\varrho_{i} R_{i} \theta, \quad R_{i}=\frac{R_{g}}{m_{i}}
$$

where $R_{g}=8.3144621$ is universal gas constant, and $m_{i}$ denotes the mollar mass of the ith specie. We can introduce the species mass fractions $Y_{1}, Y_{2}$, with $Y_{1}=\frac{\varrho_{1}}{\varrho}, Y_{2}=\frac{\varrho_{2}}{\varrho}$, it is $Y_{1}+Y_{2}=1$. The thermodynamic constants of the mixture satisfy (using the decomposition of the internal specific energy and enthalpy)

$$
\varrho c_{p}=\sum \varrho_{i} c_{p i}, \varrho c_{V}=\sum \varrho_{i} c_{V i}
$$

then the adiabatic constant $\gamma$, needed in the solution of (1), can be written as

$$
\gamma=\frac{c_{p}}{c_{V}}=\frac{\sum Y_{i} c_{p i}}{\sum Y_{i} c_{V i}}
$$


The system (1) is then extended with the conservation law of the mass for one gas component (specie)

$$
\begin{gathered}
\frac{\partial \varrho Y_{1}}{\partial t}+\frac{\partial \varrho Y_{1} v_{1}}{\partial x_{1}}+\frac{\partial \varrho Y_{1} v_{2}}{\partial x_{2}}+\frac{\partial \varrho Y_{1} v_{3}}{\partial x_{3}}= \\
\frac{\partial}{\partial x_{1}}\left(\sigma_{C} \mu_{T} \frac{\partial Y_{1}}{\partial x_{1}}\right)+\frac{\partial}{\partial x_{2}}\left(\sigma_{C} \mu_{T} \frac{\partial Y_{1}}{\partial x_{2}}\right)+\frac{\partial}{\partial x_{3}}\left(\sigma_{C} \mu_{T} \frac{\partial Y_{1}}{\partial x_{3}}\right)(2)
\end{gathered}
$$

Here $\sigma_{C}$ is diffusion coefficient. The mass conservation for the second specie is automatically satisfied via the system (1).

Here we assume the system (1),(2) equipped with the two-equation turbulent model $k-\omega$ (Kok), described in [1]. The effective turbulent viscosity is $\mu_{T}=\varrho k / \omega$.

$$
\begin{array}{r}
\frac{\partial \varrho k}{\partial t}+\frac{\partial \varrho k v_{1}}{\partial x_{1}}+\frac{\partial \varrho k v_{2}}{\partial x_{2}}+\frac{\partial \varrho k v_{3}}{\partial x_{3}}=P_{k}-\beta^{*} \varrho \omega k+ \\
+\frac{\partial}{\partial x_{1}}\left(\left(\mu+\sigma_{k} \mu_{T}\right) \frac{\partial k}{\partial x_{1}}\right)+ \\
+\frac{\partial}{\partial x_{2}}\left(\left(\mu+\sigma_{k} \mu_{T}\right) \frac{\partial k}{\partial x_{2}}\right)+\frac{\partial}{\partial x_{3}}\left(\left(\mu+\sigma_{k} \mu_{T}\right) \frac{\partial k}{\partial x_{3}}\right), \\
\frac{\partial \varrho \omega}{\partial t}+\frac{\partial \varrho \omega v_{1}}{\partial x_{1}}+\frac{\partial \varrho \omega v_{2}}{\partial x_{2}}+\frac{\partial \varrho \omega v_{3}}{\partial x_{3}}=P_{\omega}-\beta \varrho \omega \omega+ \\
+\frac{\partial}{\partial x_{2}}\left(\left(\mu+\sigma_{\omega} \mu_{T}\right) \frac{\partial \omega}{\partial x_{2}}\right)+\frac{\partial}{\partial x_{3}}\left(\left(\mu+\sigma_{\omega} \mu_{T}\right) \frac{\partial \omega}{\partial x_{1}}\right)+
\end{array}
$$

where $k$ the turbulent kinetic energy and $\omega$ the turbulent dissipation are functions of time $t$ and space coordinates $x_{1}, x_{2}, x_{3}$. The production terms $P_{k}$ and $P_{\omega}$ are given by formulas

$$
\begin{aligned}
P_{k}= & \tau_{11} \frac{\partial v_{1}}{\partial x_{1}}+\tau_{12} \frac{\partial v_{1}}{\partial x_{2}}+\tau_{21} \frac{\partial v_{2}}{\partial x_{1}}+\tau_{22} \frac{\partial v_{2}}{\partial x_{2}}+\tau_{13} \frac{\partial v_{1}}{\partial x_{3}}+ \\
& +\tau_{31} \frac{\partial v_{3}}{\partial x_{1}}+\tau_{23} \frac{\partial v_{2}}{\partial x_{3}}+\tau_{32} \frac{\partial v_{3}}{\partial x_{2}}+\tau_{33} \frac{\partial v_{3}}{\partial x_{3}}, \\
P_{\omega}= & \frac{\alpha_{\omega} \omega P_{k}}{k},
\end{aligned}
$$

where functions $\tau$ are defined in (1) with $\mu=0, \alpha_{\omega}=$ $\frac{\beta}{\beta^{*}}-\frac{\sigma_{\omega} \kappa^{2}}{\sqrt{\beta^{*}}}$ and $\sigma_{k}=\frac{2}{3}, \beta^{*}=0.09, \beta=\frac{5}{6} \beta^{*}, \sigma_{\omega}=0.5, \kappa=$ 0.41 . The cross-diffusion term $C_{D}$ is defined as

$$
C_{D}=\sigma_{d} \frac{\varrho}{\omega} \max \left\{\frac{\partial k}{\partial x_{1}} \frac{\partial \omega}{\partial x_{1}}+\frac{\partial k}{\partial x_{2}} \frac{\partial \omega}{\partial x_{2}}+\frac{\partial k}{\partial x_{3}} \frac{\partial \omega}{\partial x_{3}}, 0\right\},
$$

where $\sigma_{d}=0.5$ is constant.

\section{Numerical Method}

For the discretization of the system we proceed as described in [6]. We use either explicit or implicit finite volume method to solve the systems sequentionally. Here we present the discretization of the system (1). By $\Omega_{h}$ let us the denote the polyhedral approximation of $\Omega$. The system of the closed polyhedrons with mutually disjoint interiors $\mathcal{D}_{h}=\left\{D_{i}\right\}_{i \in J}$, where $J \subset Z^{+}=\{0,1, \ldots\}$ is an index set and $h>0$, will be called a finite volume mesh. This system $\mathcal{D}_{h}$ approximates the domain $\Omega$, we write $\bar{\Omega}_{h}=\bigcup_{i \in J} D_{i}$. The elements $D_{i} \in \mathcal{D}_{h}$ are called the finite volumes. For two neighboring elements $D_{i}, D_{j}$ we set $\Gamma_{i j}=\partial D_{i} \cap \partial D_{j}=\Gamma_{j i}$. Similarly, using the negative index $j$ we may denote the boundary faces. Here we will work with the so-called regular meshes, i.e. the intersection of two arbitrary (different) elements is either empty or it consists of a common vertex or a common edge or a common face (in 3D). The boundary $\partial D_{i}$ of each element $D_{i}$ is

$$
\partial D_{i}=\bigcup_{\Gamma_{i j} \in \Gamma_{D_{i}}} \Gamma_{i j}
$$

Here the set $\Gamma_{D_{i}}=\left\{\Gamma_{i j} ; \Gamma_{i j} \subset \partial D_{i}\right\}$ forms the boundary $\partial D_{i}$. By $\boldsymbol{n}_{i j}$ let us denote the unit outer normal to $\partial D_{i}$ on $\Gamma_{i j}$. Let us construct a partition $0=t_{0}<t_{1}<\ldots$ of the time interval $[0, T]$ and denote the time steps $\tau_{k}=t_{k+1}-t_{k}$. We integrate the system (1) over the set $D_{i} \times\left(t_{k}, t_{k+1}\right)$. With the integral form of the equations we can study a flow with discontinuities, such as shock waves, too.

$$
\begin{array}{r}
\int_{D_{i}} \int_{t_{k}}^{t_{k+1}} \frac{\partial \boldsymbol{w}}{\partial t} d x d t+\int_{t_{k}}^{t_{k+1}} \int_{D_{i}} \sum_{s=1}^{3} \frac{\partial \boldsymbol{f}_{s}(\boldsymbol{w})}{\partial x_{s}} d x d t= \\
=\int_{t_{k}}^{t_{k+1}} \int_{D_{i}} \sum_{s=1}^{3} \frac{\partial \boldsymbol{R}_{s}(\boldsymbol{w}, \nabla \boldsymbol{w})}{\partial x_{s}} d x d t
\end{array}
$$

Using the Green's theorem on $D_{i}$ it is

$$
\begin{array}{r}
\int_{D_{i}} \sum_{s=1}^{3} \frac{\partial \boldsymbol{f}_{s}(\boldsymbol{w})}{\partial x_{s}} d x=\int_{\partial D_{i}} \sum_{s=1}^{3} \boldsymbol{f}_{s}(\boldsymbol{w}) n_{s} d S \\
\int_{D_{i}} \sum_{s=1}^{3} \frac{\partial \boldsymbol{R}_{s}(\boldsymbol{w}, \nabla \boldsymbol{w})}{\partial x_{s}} d x=\int_{\partial D_{i}} \sum_{s=1}^{3} \boldsymbol{R}_{s}(\boldsymbol{w}, \nabla \boldsymbol{w}) n_{s} d S .
\end{array}
$$

Here $\boldsymbol{n}=\left(n_{1}, n_{2}, n_{3}\right)$ is the unit outer normal to $\partial D_{i}$. Further we use (5), and we rewrite (6)

$$
\begin{aligned}
\int_{D_{i}} & \left(\boldsymbol{w}\left(x, t_{k+1}\right)-\boldsymbol{w}\left(x, t_{k}\right)\right) d x+ \\
& \int_{t_{k}}^{t_{k+1}} \sum_{\Gamma_{i j} \in \Gamma_{D_{i}}} \int_{\Gamma_{i j}} \sum_{s=1}^{3}\left(\boldsymbol{f}_{s}(\boldsymbol{w})-\boldsymbol{R}_{s}(\boldsymbol{w}, \nabla \boldsymbol{w})\right)\left(n_{i j}\right)_{s} d S d t=0
\end{aligned}
$$

We define a finite volume approximate solution of the system studied (1) as a piecewise constant vector-valued functions $\mathbf{w}_{h}^{k}, k=0,1, \ldots$, where $\boldsymbol{w}_{h}^{k}$ is constant on each element $D_{i}$, and $t_{k}$ is the time instant. By $\boldsymbol{w}_{i}^{k}$ we denote the value of the approximate solution on $D_{i}$ at time $t_{k}$. We approximate the integral over the element $D_{i}$

$$
\int_{D_{i}} \boldsymbol{w}\left(x, t_{k}\right) d x \approx\left|D_{i}\right| \boldsymbol{w}_{i}^{k} .
$$

Further we proceed with the approximation of the fluxes. Usually the flux $\left.\sum_{s=1}^{3} \boldsymbol{f}_{s}(\boldsymbol{w})\left(n_{i j}\right)_{s}\right|_{\Gamma_{i j}}$ is being approximated by a numerical flux at suitable time instant $t_{l}$

$$
\left.\sum_{s=1}^{3} \boldsymbol{f}_{s}(\boldsymbol{w})\left(n_{i j}\right)_{s}\right|_{\Gamma_{i j}} \approx \boldsymbol{H}\left(\boldsymbol{w}_{i}^{l}, \boldsymbol{w}_{j}^{l}, \boldsymbol{n}_{i j}\right),
$$

with $\boldsymbol{w}_{i}^{l}, \boldsymbol{w}_{j}^{l}$ denoting the approximate solution on the elements adjacent to the edge $\Gamma_{i j}$ at the time instant $t_{l}$. In the case of a boundary face the vector $\boldsymbol{w}_{j}^{l}$ has to be specified. 
Here we show the numerical flux based on the solution of the Riemann problem for the split Euler equations. By $\boldsymbol{w}_{\Gamma_{i j}}^{l}$ let us denote the state vector $\boldsymbol{w}$ at the center of the edge $\Gamma_{i j}$ at the time instant $t_{l}$, and let us suppose $\boldsymbol{w}_{\Gamma_{i j}}^{l}$ is known. Evaluation of these values will be a question of the further analysis, here we use them to approximate the integrals with the one-point rule

$$
\int_{\Gamma_{i j}} \sum_{s=1}^{3} \boldsymbol{f}_{s}\left(\boldsymbol{w}\left(x, t_{l}\right)\right)\left(n_{i j}\right)_{s} d S \approx\left|\Gamma_{i j}\right| \sum_{s=1}^{3} \boldsymbol{f}_{s}\left(\boldsymbol{w}_{\Gamma_{i j}}^{l}\right)\left(n_{i j}\right)_{s} .
$$

$\int_{\Gamma_{i j}} \sum_{s=1}^{3} \boldsymbol{R}_{s}\left(\boldsymbol{w}\left(x, t_{l}\right), \nabla \boldsymbol{w}\left(x, t_{l}\right)\right)\left(n_{i j}\right)_{s} d S \approx\left|\Gamma_{i j}\right| \sum_{s=1}^{3} \boldsymbol{R}_{s}\left(\boldsymbol{w}_{\Gamma_{i j}}^{l}, \nabla \boldsymbol{w}_{\Gamma_{i j}}^{l}\right)\left(n_{i j}\right)_{s}$. Here $\nabla \boldsymbol{w}_{\Gamma_{i j}}^{l}$ denotes the $\nabla \boldsymbol{w}$ at the center of the edge $\Gamma_{i j}$ at time instant $t_{l}$. Now it is possible to approximate the system (8) by the following explicit finite volume scheme

$$
\begin{aligned}
& \frac{\left|D_{i}\right|}{\tau_{k}}\left(\boldsymbol{w}_{i}^{k+1}-\boldsymbol{w}_{i}^{k}\right)+ \\
& \sum_{\Gamma_{i j} \in \Gamma_{D_{i}}}\left|\Gamma_{i j}\right| \sum_{s=1}^{3}\left(\boldsymbol{f}_{s}\left(\boldsymbol{w}_{\Gamma_{i j}}^{k}\right) n_{s}-\boldsymbol{R}_{s}\left(\boldsymbol{w}_{\Gamma_{i j}}^{k}, \nabla \boldsymbol{w}_{\Gamma_{i j}}^{k}\right) n_{s}\right)=0
\end{aligned}
$$

With this finite volume formula one computes the values of the approximate solution at the time instant $t_{k+1}$, using the values from the time instant $t_{k}$, and by evaluating the values $\boldsymbol{w}_{\Gamma_{i j}}^{k}$ at the faces $\Gamma_{i j}$. In order to achieve the stability of the used method, the time step $\tau_{k}$ must be restricted by the so-called CFL condition, see [4]. The crucial problem of this discretization lies with the evaluation of the edge values $\boldsymbol{w}_{\Gamma_{i j}}^{k}$. Or one deals with the problem of finding the face fluxes $\boldsymbol{H}\left(\boldsymbol{w}_{i}^{k}, \boldsymbol{w}_{j}^{k}, \boldsymbol{n}_{i j}\right)$. It is also possible to use the implicit scheme

$$
\begin{aligned}
& \frac{\left|D_{i}\right|}{\tau_{k}} \quad\left(\boldsymbol{w}_{i}^{k+1}-\boldsymbol{w}_{i}^{k}\right)+ \\
& \quad \sum_{\Gamma_{i j} \in \Gamma_{D_{i}}}\left|\Gamma_{i j}\right|\left(\boldsymbol{H}\left(\boldsymbol{w}_{i}^{k+1}, \boldsymbol{w}_{j}^{k+1}, \boldsymbol{n}_{i j}\right)-\sum_{s=1}^{3} \boldsymbol{R}_{s}\left(\boldsymbol{w}_{\Gamma_{i j}}^{k+1}, \nabla \boldsymbol{w}_{\Gamma_{i j}}^{k+1}\right) n_{s}\right)=0
\end{aligned}
$$

The crucial problem of this discretization lies with the evaluation of the face fluxes $\boldsymbol{H}\left(\boldsymbol{w}_{i}^{k+1}, \boldsymbol{w}_{j}^{k+1}, \boldsymbol{n}_{i j}\right)$. One possibility is to use the linearization via the Taylor expansion of the vector function $\boldsymbol{H}(\boldsymbol{w} \boldsymbol{I}, \boldsymbol{w} \boldsymbol{J}, \boldsymbol{n})$, this was shown in [6]. One possibility of the face flux evaluation is to approximate the face values $\boldsymbol{w}_{\Gamma_{i j}}^{k}$ and then compute the numerical flux

$$
\left.\boldsymbol{H}\right|_{i j} ^{k}=\sum_{s=1}^{3} \boldsymbol{f}_{s}\left(\boldsymbol{w}_{\Gamma_{i j}}^{k}\right)\left(n_{i j}\right)_{s} .
$$

To approximate the face values $\boldsymbol{w}_{\Gamma_{i j}}^{k}$ at time instant $t_{k}$ we solve the simplified system (14) in the vicinity of the face $\Gamma_{i j}$ in time with the initial condition formed by the state vectors $\boldsymbol{w}_{i}^{k}$ and $\boldsymbol{w}_{j}^{k}$. Using the rotational invariance of the Euler equations, the system is expressed in a new Cartesian coordinate system $\tilde{x}_{1}, \tilde{x}_{2}, \tilde{x}_{3}$ with the origin at the center of the gravity of $\Gamma_{i j}$ and with the new axis $\tilde{x}_{1}$ in the direction of $\boldsymbol{n}=\left(n_{1}, n_{2}, n_{3}\right)$, given by the face normal $\boldsymbol{n}=\boldsymbol{n}_{i j}$. Then the derivatives with respect to $\tilde{x}_{2}, \tilde{x}_{3}$ are neglected and we get the so-called split 3D Euler equations, see [4, page 138]:

$$
\frac{\partial \boldsymbol{q}}{\partial t}+\frac{\partial \boldsymbol{f}_{1}(\boldsymbol{q})}{\partial \tilde{x}_{1}}=0
$$

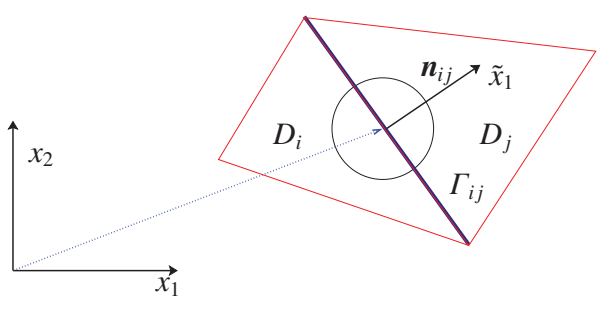

Fig. 1. Coordinate transformation for the inner edges in 2D.

The values $\boldsymbol{w}_{i}^{k}$ and $\boldsymbol{w}_{j}^{k}$ adjacent to the face $\Gamma_{i j}$ are known, forming the initial conditions

$$
\begin{array}{ll}
\boldsymbol{q}\left(\tilde{x}_{1}, 0\right)=\boldsymbol{q}_{L}=\mathbb{Q} \boldsymbol{w}_{i}^{k}, & \tilde{x}_{1}<0, \\
\boldsymbol{q}\left(\tilde{x}_{1}, 0\right)=\boldsymbol{q}_{R}=\mathbb{Q} \boldsymbol{w}_{j}^{k}, & \tilde{x}_{1}>0 .
\end{array}
$$

The transformation matrix $\mathbb{Q}$ is defined in [6]. The problem (14), (15), (16) has a unique "solution" in $(-\infty, \infty) \times$ $(0, \infty)$, the analysis can be found in [4, page 138]. Let $\boldsymbol{q}_{R S}\left(\boldsymbol{q}_{L}, \boldsymbol{q}_{R}, \tilde{x}_{1}, t\right)$ denote the solution of this problem at the point $\left(\tilde{x}_{1}, t\right)$. We are interested in the solution of this local problem at the time axis, which is the sought solution in the local coordinates $\boldsymbol{q}_{\Gamma_{i j}}=\boldsymbol{q}_{R S}\left(\boldsymbol{q}_{L}, \boldsymbol{q}_{R}, 0, t\right)$. The backward transformation of the state vector $\boldsymbol{q}_{\Gamma_{i j}}$ into the global coordinates is

$$
\boldsymbol{w}_{\Gamma_{i j}}^{k}=\mathbb{Q}^{-1} \boldsymbol{q}_{\Gamma_{i j}}=\mathbb{Q}^{-1} \boldsymbol{q}_{R S}\left(\mathbb{Q} \boldsymbol{w}_{i}^{k}, \mathbb{Q} \boldsymbol{w}_{j}^{k}, 0, t\right) .
$$

The constructed numerical flux can be written as

$$
\boldsymbol{H}(\boldsymbol{u}, \boldsymbol{v}, \boldsymbol{n}):=\sum_{s=1}^{3} \boldsymbol{f}_{s}\left(\mathbb{Q}^{-1} \boldsymbol{q}_{R S}(\mathbb{Q} \boldsymbol{u}, \mathbb{Q} \boldsymbol{v}, 0, t)\right) n_{s} .
$$

Let $\Gamma_{i j}$ be the face of the element $D_{i}$ laying at the boundary of the computational area. To approximate the face values $\boldsymbol{w}_{\Gamma_{i j}}^{k}$ at time instant $t_{k}$ we solve the incomplete simplified system (14) in the vicinity of the face $\Gamma_{i j}$ in time with the initial condition (15) determined by the state vector $\boldsymbol{w}_{i}^{k}$. By adding properly chosen equations into the system (14),(15) it is possible to reconstruct the boundary state $\boldsymbol{q}_{B}$ such that the system (14),(15) has a unique solution at the boundary, see [3]. We will refer to these added equations as to complementary conditions. Several choices of the complementary conditions were discussed in [3], [5].

\section{Examples}

All the results were obtained with the own-developed codes. Here we present simple example of the flow in the cube in $3 \mathrm{D}$, the simulation was made for the mixture of the dry air and the water steam. All the used constants and input data are given in SI units. For this special case we used the constants $\sigma_{C}=1 / 1.3$,

\begin{tabular}{c|c|c} 
& dry air & water steam \\
\hline$c_{p}, J K^{-1} \mathrm{~mol}^{-1}$ & 1004 & 1911 \\
$c_{V}, J K^{-1} \mathrm{~mol}^{-1}$ & 712 & 1450 \\
$\gamma=\frac{c_{p}}{c_{V}}$ & 1.401123 & 1.317931 \\
$R=c_{p}-c_{V}, J K^{-1} \mathrm{~mol}^{-1}$ & 292 & 461
\end{tabular}

The initial condition was formed by the whole area filled with the dry air, velocity regime $v=\left(15 \mathrm{~m} \mathrm{~s}^{-1}, 0 \mathrm{~m} \mathrm{~s}^{-1}, 10 \mathrm{~m} \mathrm{~s}^{-1}\right)$. 
At the inlet we consider conservation of the total quantities $\theta_{0}=273.15 \mathrm{~K}, p_{0}=101325 \mathrm{~Pa}$, and the velocity direction $\boldsymbol{d}=(15,0,10)$. At the outlet part we prefered the average pressure $p=101115.15 \mathrm{~Pa}$. At the chosen part of the inlet (left) boundary we applied the maximal concentration $(=1)$ of the added gas specie (water steam). This source of admixture flows into the area and dissipates. The computational mesh and resulting isolines of mixture concentration can be seen in figure 2, the figure 3 shows the isosurfaces of density.
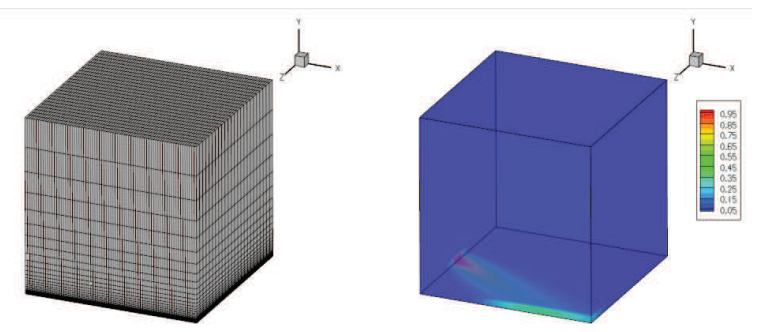

Fig. 2. The passive mixture simulation, used mesh and concentration of added gas component.

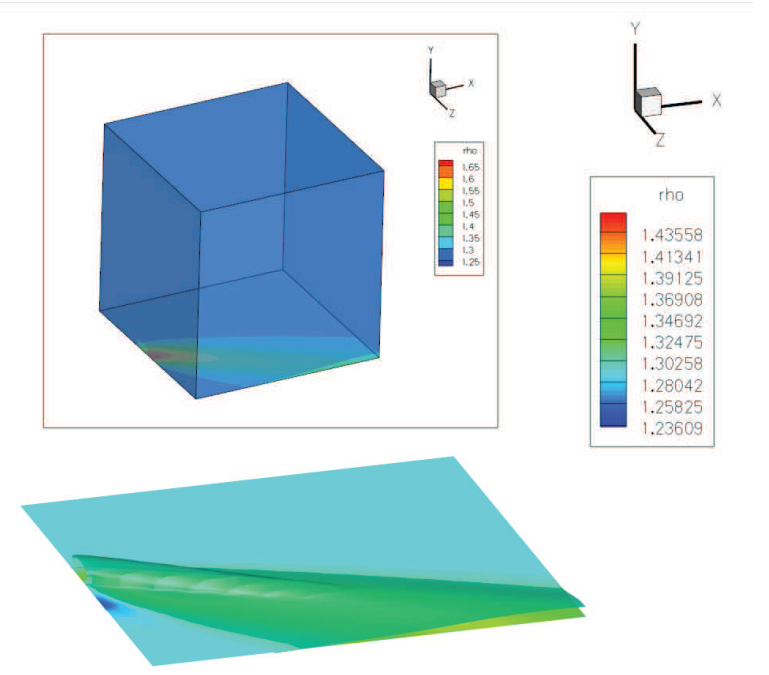

Fig. 3. The passive mixture simulation, isolines of density.

Another example simulates the emission of gas with enhanced total quantities into the area. We simulate the injected gas with the total pressure $p_{o}=101325+12882.888 \mathrm{~Pa}$ total density $\varrho_{o}=2.4955963 \mathrm{~kg} \mathrm{~m}^{-3}$, and properties $R_{1}=$ 287.3 $\mathrm{JK}^{-1} \mathrm{~mol}^{-1}, \gamma_{1}=1.3018$, gas is emited through the chosen part of the inlet boundary. The initial condition was formed by the whole area filled with air, velocity regime $15 \mathrm{~m} \mathrm{~s}^{-1}$. The simulation was made in 2D, figure 4 demonstrates the density evolution in time.
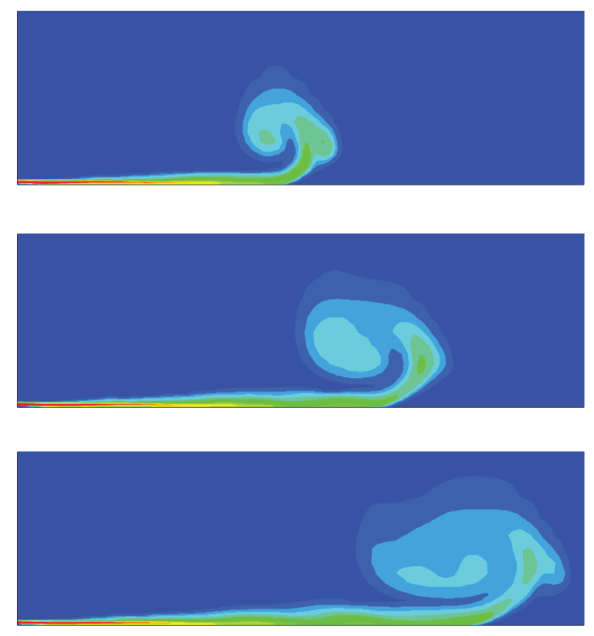

Fig. 4. The passive mixture simulation, isolines of density.

\section{Conclusion}

This paper shows the formulation of the equations describing the mixture of two inert perfect gases in 3D. The numerical method (finite volume method) is applied for the solution of these equations. Own software was programmed. The modification of the Riemann problem is used at the boundaries.

\section{Acknowledgment}

This result originated with the support of Ministry of the Interior of the Czech Republic, project SCENT. The authors acknowledge this support.

\section{References}

1. C. J. Kok. AIAA Journal, Vol. 38., No. 7., (2000).

2. M. Kyncl and J. Pelant. Applications of the NavierStokes equations for $3 d$ viscous laminar flow for symmetric inlet and outlet parts of turbine engines with the use of various boundary conditions. Technical report R3998, VZLÚ, Beranových 130, Prague, (2006).

3. M. Kyncl. Numerical solution of the three-dimensional compressible flow. Master's thesis, Prague, (2011). Doctoral Thesis.

4. M. Feistauer, J. Felcman, and I. Straškraba. Mathematical and Computational Methods for Compressible Flow. Oxford University Press, Oxford, (2003).

5. M. Kyncl and J. Pelant. Implicit method for the $3 d$ Euler equations. Technical report R5375, VZLÚ, Beranových 130, Prague, 2012.

6. M. Kyncl and J. Pelant. Implicit method for the $3 d$ RANS equations with the $k-w$ (Kok) Turbulent Model. Technical report R5453, VZLÚ, Beranových 130, Prague, 2012. 